\title{
Fractura de cuerpos cavernosos. Serie de casos.
}

\author{
Martínez Ruiz J, Pastor Navarro H, Carrión López P, Giménez Bachs JM, Donate Moreno MJ, \\ Virseda Rodríguez JA.
}

Servicio de Urología. Complejo Hospitalario Universitario de Albacete.

Actas Urol Esp. 2008;32(6):599-602

\section{RESUMEN}

FRACTURA DE CUERPOS CAVERNOSOS. SERIE DE CASOS

La rotura de cuerpos cavernosos en una entidad infrecuente encuadrada dentro de las urgencias urológicas, con una presentación típica y que requiere generalmente de una actuación quirúrgica precoz para evitar alteraciones funcionales y estéticas.

Exponemos los pacientes atendidos por rotura de cuerpos cavernosos en el Servicio de Urgencias del Complejo Hospitalario Universitario de Albacete durante los años 1997 a 2007, analizando su forma de presentación y el mecanismo u origen de la lesión, el tratamiento recibido y las complicaciones surgidas.

Palabras clave: Cuerpos cavernosos. Rotura. Pene. Complicaciones.

\section{ABSTRACT}

FRACTURE OF THE PENIS: A CASE SERIES

Penile fracture is an uncommon urological emergency with a typical presentation, which needs early surgery to avoid aesthetic and functional sequelaes.

We have analyzed all the patients with a discharge diagnosis of fractures penis among 1997 to 2007 , attended in the emergency service of Complejo Hospitalario Universitario de Albacete, showing its clinical features, attributed mechanism of injury, treatment and main complications.

Keywords: Penis. Rupture. Complications.

$\mathrm{L}$ a rotura de cuerpos cavernosos es una entidad cuya frecuencia real se desconoce a pesar de tener consecuencias morfológicas y funcionales graves $^{1}$. La principal causa de que muchos de los casos no se encuentren documentados se debe a que bastantes pacientes no consultan por ser una situación embarazosa, que según nuestra experiencia hace la anamnesis dificultosa, encontrando serias dificultades para que el paciente revele el verdadero origen de la lesión ${ }^{2}$.

Las lesiones traumáticas del pene son variadas. Pueden deberse tanto a traumatismos abiertos como cerrados. La patología más frecuente es la ruptura de cuerpos cavernosos por traumatismos no penetrantes ${ }^{3}$.

Presentamos una serie de cinco casos acontecidos en nuestro centro durante los últimos diez años.

\section{MATERIAL Y MÉTODOS}

Se estudiaron los pacientes con diagnóstico de rotura de cuerpos cavernosos desde enero de 1997 a diciembre de 2007, atendidos en el Complejo Universitario Hospitalario de Albacete.

La edad, tiempo de demanda de atención, causa de la lesión, síntomas y signos, lugar de rotura, hallazgos intraoperatorios y secuelas fueron analizados y descritos.

\section{RESULTADOS}

Se analizaron 5 pacientes. La media de edad fue de 37 años (rango 27-51 años). El intervalo de tiempo desde que se produjo la lesión hasta la solicitud de atención fue de 6 horas a 3 días.

Dos casos fueron atribuidos a masturbación, dos casos se debieron a coito intempestivo y uno a traumatismo cerrado mientras dormía al rodar sobre el pene en la cama. 
Todos los pacientes refirieron la audición de un chasquido como desencadenante de la clínica y cuatro de ellos dolor acompañante y hematoma. Sólo dos de ellos refirieron desviación peneana como signo característico del cuadro.

En tres casos se realizó una ecografía como prueba complementaria hallando en dos de ellas lesión hipoecoica que alteraba la continuidad de la túnica albugínea (Fig. 1). En todos los casos la rotura fue de uno de los cuerpos cavernosos, (cuatro del derecho y uno del izquierdo). En dos pacientes la rotura fue en el tercio proximal, tres en la porción media y ninguna en el tercio distal. Todos los pacientes fueron sometidos a tratamiento quirúrgico precoz. Mediante incisión subcoronal se realizó denudación hasta la base del pene, evacuación del hematoma y sutura del cuerpo cavernoso roto con material reabsorbible con puntos discontinuos. La duración de la hospitalización fue de 1 a 3 días. No existe seguimiento de uno de los pacientes por no acudir a las citas programadas, y en el resto, el rango de seguimiento oscila entre uno y ocho meses. Sólo uno de los pacientes presenta una discreta fibrosis en el lado de la lesión sin incurvación peneana.

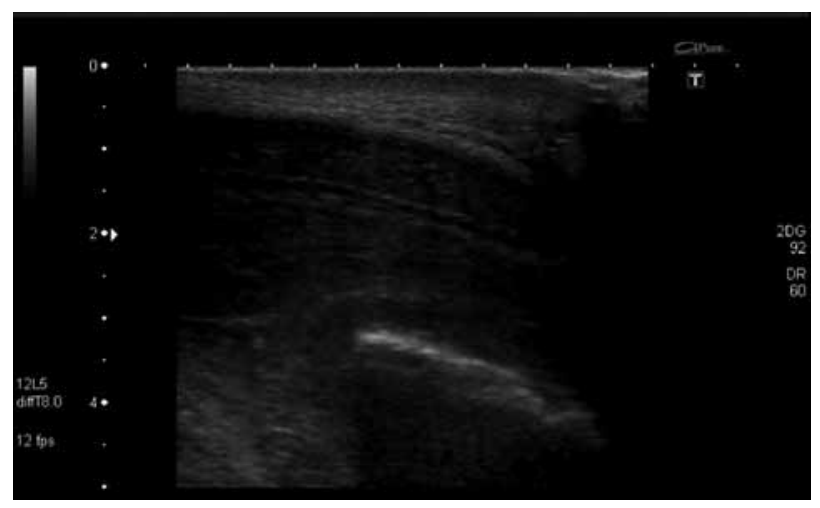

FIGURA 1. Ecografía del pene. Lesión hipoiecoica que altera la continuidad de la túnica albuginea.

\section{DISCUSIÓN}

La rotura de los cuerpos cavernosos es un cuadro relativamente poco frecuente que se debe fundamentalmente a un traumatismo sobre el pene en erección ${ }^{1}$.

La túnica albugínea tiene un grosor de $2 \mathrm{~mm}$ en estado flácido, adelgazando hasta 0,5-0,25 mm durante la erección ${ }^{4,5}$. La disminución del espesor junto al descenso de la elasticidad de dicha túnica y al aumento de la presión intracavernosa (por encima de $1.500 \mathrm{~mm} \mathrm{Hg}$ ) hacen que el pene sea menos resistente a la angulación ${ }^{4,6}$.
El mecanismo más frecuente de rotura es el coito (33-58\%), debido a la contusión del pene sobre el periné o pubis de la pareja. Otros mecanismos son la masturbación, caídas o cambios de posición en la cama con el pene erecto, etc ${ }^{1,4,7}$.

El diagnóstico se basa en la historia clínica y la exploración física ${ }^{7}$. Si aún así existen dudas se pueden utilizar diversos métodos diagnósticos complementarios. La cavernosografía en una técnica invasiva que no se suele disponer en urgencias y que puede dar lugar a fibrosis cavernosa e infección como principal complicación. Otra prueba complementaria es la ecografía, prueba no invasiva pero que no permite detectar pequeñas roturas, aunque es útil para ver el hematoma. La resonancia magnética (RM) nos permite examinar la porción pendular del pene, especialmente la túnica albugínea, pero su elevado coste y escasa accesibilidad en urgencias la hacen una prueba poco rentable. Por último la uretrocistografía miccional es una prueba útil que debe realizarse cuando se sospecha lesión de la uretra ${ }^{4}$.

El traumatismo peneano suele acompañarse de la audición de un chasquido seguido de detumescencia rápida, dolor o no, incurvación del pene hacia el lado contrario a la fractura y aparición de hematoma genital, que será exclusivamente peneano si la fascia de Buck mantiene su integridad; mientras que será inguinoescrotal cuando es contenido por la fascia de de Colles ${ }^{3,7}$. La fractura proximal es más frecuente, y en el caso de que el agente responsable sea el coito, la localización será ventral. Igualmente la rotura del cuerpo cavernoso derecho es más frecuente que la del izquierdo, como en nuestra serie. No existe una clara explicación que justifique esta diferencia de afectación. La aparición de uretrorragia, hematuria macroscópica, dificultad miccional o retención urinaria sugieren lesión uretral, que en caso de aparecer deberá repararse y añadir profilaxis antibiótica. En caso de que el hematoma sea de gran cuantía y comprima el cuerpo esponjoso, puede aparecer dificultad miccional aun estando íntegra la uretra. Ninguno de nuestros pacientes tuvo lesión de la uretra.

El diagnóstico diferencial se establece con la lesión de la vena dorsal del pene, que se debe también a un traumatismo y la sintomatología es similar, aunque no suelen presentar chasquido ni desviación peneana ${ }^{4}$. 
El tratamiento de la rotura de los cuerpos cavernosos ha ido evolucionando a lo largo de los años. La tendencia actual que demuestra tener menor incidencia de secuelas es la reparación quirúrgica inmediata, pero parece que algunos pacientes no se benefician claramente de este tipo de intervención, y pueden tener similares resultados con tratamiento conservador mediante vendaje compresivo y frío, antiinflamatorios, antiandrógenos y antibióticos y/o cirugía diferida ${ }^{8}$. El principal problema estriba en discriminar qué pacientes son susceptibles de tratamiento quirúrgico inmediato y cuales no. Parece que los que claramente se benefician de la cirugía no diferida son aquellos con lesión uretral, dificultad para la micción y gran hematoma ${ }^{8}$.

El abordaje quirúrgico más extendido es la incisión subcoronal circunferencial y la denudación del pene hasta la base; lo que nos permite una adecuada exposición y exploración de los cuerpos cavernosos y de la uretra, practicando sutura discontinua de la túnica albugínea con material reabsorbible de 3/0 o 4/0 (Fig. 2); aunque se obtienen resultados parecidos con material no reabsorbible con puntos invertidos, ya que al no estar el nudo en contacto con la superficie del pene se reduce la sensación de dureza marcadamente puesta de manifiesto con las relaciones sexuales (sensación que se asocia a este tipo de sutura cuando no se invierte el nudo) ${ }^{5,6}$. Se debe reservar especialmente la sutura no reabsorbible para los raros casos de rotura recidivante ${ }^{5}$.

Otro tipo de abordaje consiste en la realización de una incisión corporal longitudinal sobre la zona del hematoma, ideal cuando sospechamos lesiones albugíneas pequeñas, a pesar de no permitirnos la exploración del resto del pene pudiendo pasar por alto lesiones acompañantes. Existe otro tipo de abordaje descrito basado en la incisión inguinoescrotal, que al trabajar sobre una zona no edematosa permite una mejor visualización de la rotura, aunque este tipo de incisión tampoco permite la evaluación de lesiones asociadas ${ }^{7}$.

Las complicaciones más frecuentes de la rotura de cuerpos cavernosos, sobre todo en casos no seleccionados con manejo conservador, son: incurvación peneana, erecciones dolorosas, fístulas uretrocutánea, fístula cavernosa, infección, placa fibrótica, estenosis e impotencia ${ }^{9}$.

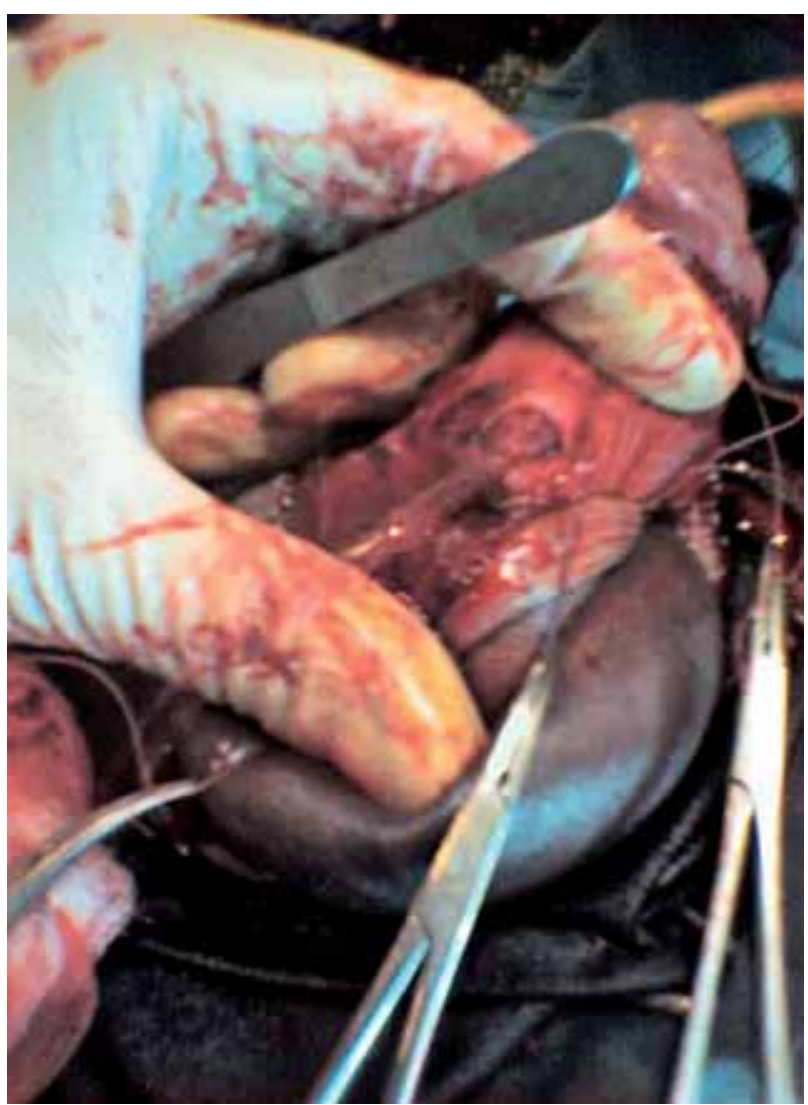

FIGURA 2. Imagen de uno de nuestros pacientes. Reparación quirúrgica precoz mediante sutura con puntos sueltos del cuerpo cavernoso roto.

El diagnóstico precoz y la reparación quirúrgica temprana contribuyen a disminuir la hospitalización, las secuelas y facilitar la recuperación satisfactoria de la actividad sexual ${ }^{5,6,8-10}$.

\section{CONCLUSIÓN}

La rotura de cuerpos cavernosos es una rara entidad encuadrada dentro de las urgencias urológicas con clínica típica que facilita el diagnóstico.

La cirugía inmediata con reparación de la albugínea con puntos sueltos de material reabsorbible es el tratamiento de elección porque permite una recuperación funcional rápida con disminución de las secuelas; sin embargo, se pueden obtener similares resultados en cuanto a complicaciones con sutura no reabsorbible con puntos invertidos. En casos seleccionados (sin lesión uretral ni gran hematoma) puede optarse por tratamiento conservador y/o cirugía diferida. 


\section{REFERENCIAS}

1. De Rose AF, Giglio M, Carmignani G. Traumatic rupture of the corpora cavernosa: new physiopathologic acquisitions. Urology. 2001;57(2):319-322.

2. Fergany AF, Angermeier KW, Montague DK. Review of Cleveland clinic experience with penile fracture. Urology. 1999;54(2):352355 .

3. Tejido Sánchez A, Martín Muñoz MP, Villacampa Abuá F, de la Morena Gallego JM, Suárez Charneco A, Leiva Galvis O. Tratamiento quirúrgico de la fracturo de pene. Nuestra experiencia. Actas Urol Esp. 1999:23(9):784-788.

3. Tejido Sánchez A, Martín Muñoz MP, Villacampa Abuá F, de la Morena Gallego JM, Suárez Charneco A, Leiva Galvis O. Surgical management of the penile fractures. Our experience. Actas Urol Esp. 1999:23(9):784-788.

4. Lorenzo Romero JG, Segura Martín M, Salinas Sánchez M, Pastor Guzmán JM, Hernández Millán I, Martínez Martín M, et al. Rotura de cuerpos cavernosos: evaluación y manejo terapéutico. Actas Urol Esp. 1999;23(7):635-639.

5. Paparel P, Ruffion A. Rupture des corps caverneux: aspects techniques de la prise en charge. Annales d`urologie. 2006; 40 (4):267-272.
6. Jack GS, Garraway I, Reznichek R, Rajfer J. Current Treatment Options for Penile Fractures. Rev Urol. 2004;6(3):114-120.

7. Rodríguez Alonso A, González Blanco A, Cespón Outeda E, Bonelli Martín C, Lorenzo Franco J, Cuerpo Pérez MA et al. Fractura de pene. Aportación de un caso. Actas Urol Esp. 2001:25 (10);765-769.

8. Muentener M, Suter S, Hauri D, Sulser T. Long-term experience with surgical and conservative treatment of penile fracture. J Urol. 2004; 172(2):576-579.

9. Mydlo JH. Surgeon experience with penile fracture. J Urol. 2001:166(2):526-529.

10. Eke N. Fracture of the penis. Br J Surg. 2002;89(5):555-565.

Correspondencia autor: Dr. J. Martínez Ruiz Servicio de Urología

Complejo Hospitalario Universitario de Albacete

Hermanos Falcó, 37 - 02006 Albacete

E-mail autor: jesus.martinez.ruiz@gmail.com

Información artículo: Original - Urología femenina

Trabajo recibido: febrero 2008

Trabajo aceptado: marzo 2008 